\title{
On the Left-/Right-Branching Asymmetry in Mandarin Tone 3 Sandhi
}

\author{
Fulang Chen \\ Massachusetts Institute of Technology
}

\section{Introduction}

Mandarin (Chinese) is a tonal language with four distinctive tones: T1, T2, T3, and T4. In (1), the digits alongside the pinyin transliterations (jiao) represent the pitch values of each tone; in the traditional Chao (1930) notation 5 indicates the top of the pitch space and 1 the bottom.

(1) Tones in Mandarin (Yip 2002)

\begin{tabular}{|c|c|c|c|}
\hline T1 & H & jiao55 & 'teach' \\
\hline T2 & LH & jiao35 & 'chew' \\
\hline T3 & L(LH) & jiao21(4) & 'mix' \\
\hline T4 & HL & jiao53 & 'call' \\
\hline
\end{tabular}

T3 has three variants: it is low falling-rising (LLH) in citation form and pre-pausally, (mid-)rising (LH) before another T3, and low(-falling) (L) elsewhere (Chao 1948, 1968). The underlying representation of T3 is controversial: Chao $(1948,1968)$ assumes that the $\mathrm{L}$ tone variant is derived from the dipping tone (LLH). In this paper, I assume, following Yip $(1980,2002)$, that T3 is underlyingly L.

T3 Sandhi (T3S) is a phonological process by which a T3 is changed into a sandhi tone $(s)$, which has the phonological characteristics of T2, when it is followed by another T3; according to Yip (1980, 2002), it is a dissimilatory process where a $\mathrm{H}$ tone is inserted between two $\mathrm{L}$ tones, as schematized in (2) and illustrated by the expression in (3).

(2) $\mathrm{T} 3 \mathrm{~S}$ in Mandarin

$\mathrm{L} \quad \mathrm{LH} \quad \mathrm{L}(\mathrm{LH})$

$3 \rightarrow s /$ - 3

(3) 'good wine'

$\begin{array}{lll}\text { UR: } & \text { hao3 } & \text { jiu3 } \\ & \text { good } & \text { wine } \\ \text { SR: } & s & 3\end{array}$

T3S is a domain-sensitive phenomenon. When more than two successive T3 syllables occur, three distinct patterns of realization are generally recognized in the literature. First, grammatically unstructured strings of T3 syllables such as the number wu3 'five' are assumed to be grouped into Minimal Rhythm Units (MRUs) that consist of two or three syllables, as shown in (4) (Chen 2000). T3S applies within each MRU; e.g., the rhythmic grouping gives rise to an alternating sandhi pattern for a string of four T3 syllables, as shown in (4b). Note that in MRUs that consist of three T3 syllables, all but the rightmost T3 is changed into the sandhi tone.

\footnotetext{
* I would like to thank Michael Kenstowicz for his patient guidance and constructive advice on this research work. For feedback and comments, I am also grateful to the participants of the Fall 2019 Workshop in Linguistics at MIT, reviewers and audience at the 2020 Annual Meeting on Phonology (AMP 2020) and the 51st Annual Meeting of the North East Linguistic Society (NELS 51), Danfeng Wu, Daniel Asherov, David Pesetsky, Donca Steriade, Edward Flemming, Jennifer Bellik, Norvin Richards, and Suzanne Flynn.
} 
(4)

\begin{tabular}{|ll|c|}
\hline & Underlying representation & Surface representation \\
\hline a. & $\begin{array}{l}w u 3 \text { wu3 } w u 3 \\
\text { 'five five five' }\end{array}$ & $(s s 3)$ \\
\hline b. $\begin{array}{l}w u 3 w u 3 w u 3 w u 3 \\
\text { 'five five five five' }\end{array}$ & $(s 3)(s \quad 3)$ \\
\hline c. $\begin{array}{l}w u 3 \text { wu3 } w u 3 w u 3 w u 3 \\
\text { 'five five five five five' }\end{array}$ & $(s 3)(s s 3)$ \\
\hline
\end{tabular}

The second and third patterns of realization for a sequence of T3 syllables depend on the syntactic structure of the expression. Cheng $(1970,1973)$, among others, observes a left-/right-branching asymmetry in T3S: Expressions that have a left-branching syntactic structure only have a non-alternating sandhi pattern in which all but the rightmost T3 is changed to the sandhi tone, as in (5a) and (6a). Note that the rhythmic grouping seen in (4b) is not possible with a left-branching expression, as shown in (6b).

\begin{tabular}{lllll}
\multicolumn{4}{l}{ 'leave a bit earlier' } \\
UR: $\quad$ [VP & [AP & zao3 & dian3] & zou3 $]$ \\
& & early & a bit & leave \\
SR: & & $s$ & $s$ & 3 \\
SR: & & $* 3$ & $s$ & 3
\end{tabular}

(6) 'It is good to leave a bit earlier.'

\begin{tabular}{|c|c|c|c|}
\hline UR: $\quad[\mathrm{IP} \quad[\mathrm{VP} \quad[\mathrm{AP}$ & $\begin{array}{l}\text { zao3 } \\
\text { early }\end{array}$ & $\begin{array}{l}\operatorname{dian} 3] \\
\text { a bit }\end{array}$ & $\begin{array}{l}z o u 3] \\
\text { leave }\end{array}$ \\
\hline SR: & $s$ & $s$ & $s$ \\
\hline SR: & $*_{S}$ & 3 & s \\
\hline SR: & $* 3$ & $s$ & $s$ \\
\hline
\end{tabular}

By contrast, the pattern of realization of expressions that have a right-branching syntactic structure is more variable. Its analysis is more challenging and will be the major focus of this paper. As exemplified below, the expression in (7) has an alternating sandhi pattern at slow speech, and a non-alternating sandhi pattern at fast speech. The expression in (8) has three possible sandhi patterns.

\begin{tabular}{|c|c|c|c|}
\hline UR: & {$\left[\begin{array}{cc}\text { [vp } & \text { mai3 } \\
\text { buy }\end{array}\right.$} & {$\left[\begin{array}{ll}\text { [NP } & \text { hao3 } \\
& \text { good }\end{array}\right.$} & $\begin{array}{l}\text { jiu3]] } \\
\text { wine }\end{array}$ \\
\hline SR: & 3 & $s$ & 3 \\
\hline SR: & $s$ & $S$ & 3 \\
\hline
\end{tabular}

(8) 'want to buy good wine'

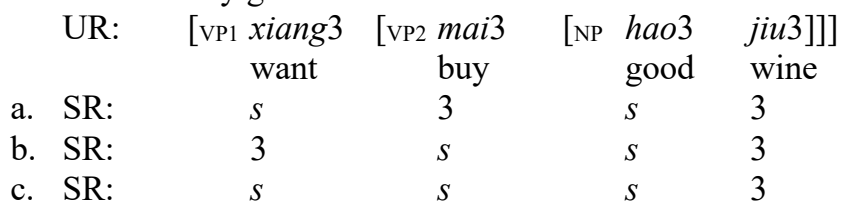

In this paper, I will pursue the hypothesis that $\mathrm{T} 3 \mathrm{~S}$ applies cyclically bottom-up on a prosodic structure matched from the syntactic structure of an expression, along the lines of the Match Theory of syntacticprosodic constituency correspondence (Selkirk 2011). Specifically, I will propose to account for the left/right-branching asymmetry in T3S by the interaction of Match Phrase constraints, which require the prosodic structure to be isomorphic to the syntactic structure, and Strong Strong Start, which is a more restricted version of Selkirk's (2011) Strong Start constraint. The effect of Strong Strong Start is that a right-branching syntactic structure is altered to a prosodic structure that has equal-sisters constituents in the sense of Myrberg (2013), either by flattening the recursive syntactic structure to create a flat, equal-sisters prosodic structure, or by grouping syntactic non-sisters at the left edge to create a recursive, equal-sisters 
prosodic structure. The interaction of Match Phrase constraints and Strong Strong Start will predict that different prosodic structures are possible outputs for a right-branching expression, while for a left-branching expression the only possible output is a left-branching prosodic structure. I will show that the various possible sandhi patterns for a right-branching expression and the non-alternating sandhi pattern for a left-branching expression can be derived when T3S applies cyclically bottom-up on the proposed prosodic structures.

The rest of the paper is organized as follows: in section 2, I present evidence that T3S is a cyclic process and that $\mathrm{T} 3 \mathrm{~S}$ applies on a prosodic structure distinct from the syntactic structure of an expression; in section 3, I propose a Match-Theory analysis of the left-/right-branching asymmetry in T3S; section 4 provides an OT analysis of the T3S process that is compatible with the prosodic structures proposed in section 3; section 5 concludes.

\section{Cyclic application and prosodic structure}

This section is dedicated to presenting evidence that T3S is a cyclic process and that $\mathrm{T} 3 \mathrm{~S}$ applies on a prosodic structure distinct from the syntactic structure of an expression. Cheng $(1970,1973)$, among others, argues that T3S is a cyclic process. He proposes that whether T3S applies between two successive T3 syllables is determined by the depths of syntactic boundaries and speech rate. Syntactic depths are indicated by the numbers $\underline{1}, \underline{2}, \underline{3}$, etc.: the number between syllables that are syntactic sisters is $\underline{1}$; the numbers between subsequent sister constituents in the syntactic structure are $\underline{2}, \underline{3}$, etc. In the right-branching example in (9), the syntactic depth between hao3 'good' and jiu 3 'wine' is 1 ; the syntactic depth between mai3 'buy' and the NP is 2 . Because the alternating sandhi pattern in (9a) and the non-alternating sandhi pattern in (9b) correlate with speech rate, Cheng suggests that T3S is blocked by deep syntactic boundaries (indicated by \#) at slow speech, while T3S applies across deep syntactic boundaries at fast speech.

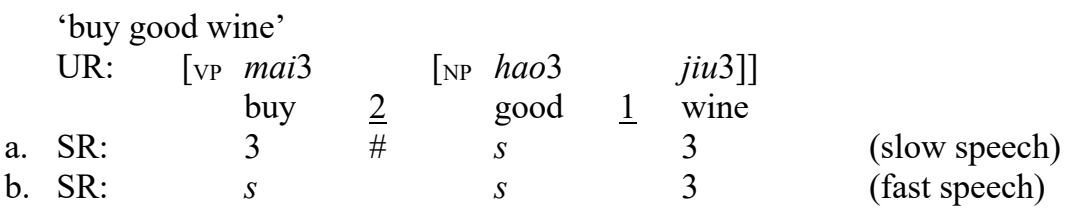

In the left-branching example in (10) and the right-branching example in (11), T3S cannot apply between dian3 'a bit' and zou3 'leave', as in (10a), or between xiang3 'want' and mai3 'buy', as in (11a), if it is blocked by deep syntactic boundaries, contrary to fact. To confront this problem, Cheng proposes that reapplication of the T3S process is necessary to eliminate any adjacent $\mathrm{T} 3$ syllables in (10a) and (11a), giving rise to the non-alternating sandhi pattern in $\left(10 a^{\prime}\right)$, and the alternating sandhi pattern in $\left(11 a^{\prime}\right)$, respectively.

\begin{tabular}{|c|c|c|c|c|c|}
\hline UR: & {$\left[\begin{array}{lll}\mathrm{VP} & {[\mathrm{AP}} & z a o 3\end{array}\right.$} & & dian3] & & zou3] \\
\hline & early & $\underline{1}$ & a bit & $\underline{2}$ & leave \\
\hline SR: & $s$ & & 3 & \# & 3 \\
\hline SR: & $s$ & & $s$ & & 3 \\
\hline SR: & $s$ & & $s$ & & 3 \\
\hline
\end{tabular}

\begin{tabular}{|c|c|c|c|c|c|c|c|c|}
\hline \multirow[t]{2}{*}{ UR: } & [VP1 xiang3 & & [VP2 mai3 & & {$[\mathrm{NP}$} & hao3 & & jiu3]]] \\
\hline & want & $\underline{3}$ & buy & $\underline{2}$ & & good & $\underline{1}$ & wine \\
\hline SR: & 3 & \# & 3 & \# & & $s$ & & 3 \\
\hline SR: & $s$ & & 3 & & & $s$ & & 3 \\
\hline SR: & 3 & \# & $s$ & & & $s$ & & 3 \\
\hline SR: & $s$ & & $s$ & & & $s$ & & 3 \\
\hline
\end{tabular}

Cheng's approach to the left-/right-branching asymmetry can be captured by assuming that T3S applies cyclically bottom-up, from syllables that are syntactic sisters to syllables in subsequent syntactic constituents in the syntactic structure of an expression. Cyclic, bottom-up application of the T3S process ensures that a 
left-branching expression has a non-alternating sandhi pattern, while a right-branching expression has an alternating sandhi pattern. The other possible sandhi patterns for a right-branching expression can be derived assuming the initial cycle coincides with a larger syntactic constituent, e.g., VP2 for (11b) and VP1 for (11c).

Kaisse (1985) discusses examples like (12), which has the possible output (12b), in which T3S does not apply between linearly adjacent but structurally non-adjacent $\mathrm{T} 3$ syllables, li3 'Li' and mai3 'buy'. From (12b), it is clear that the NP and the VP constitute different sandhi domains.

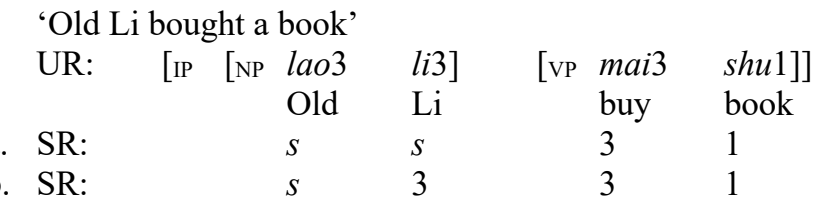

Shih (1986, 1997) and Chen (2000) observe that a right-branching expression such as (13) has the sandhi pattern in (13b), indicating that syntactic non-sisters, xiang3 'want' and mai3 'buy', can form a sandhi domain.

(13) 'want to buy good book'

\begin{tabular}{|c|c|c|c|c|c|}
\hline UR: & $\begin{array}{c}\text { [V1 } \text { xiang3 } \\
\text { want }\end{array}$ & $\begin{array}{c}\text { [vP2 mai3 } \\
\text { buy }\end{array}$ & {$[\mathrm{NP}$} & $\begin{array}{l}\text { hao3 } \\
\text { good }\end{array}$ & $\begin{array}{l}\text { shu1]]] } \\
\text { book }\end{array}$ \\
\hline a. SR: & $s$ & $s$ & & 3 & 1 \\
\hline b. SR: & $S$ & 3 & & 3 & 1 \\
\hline
\end{tabular}

Thus, they argue that T3S applies on a prosodic structure that is distinct from the syntactic structure of an expression. In particular, the right-branching syntactic structure in (13) must have a corresponding prosodic structure where xiang3 'want' and mai3 'buy' are sisters.

\section{A Match-Theory analysis}

Taking into account the evidence presented in section 2, I will assume that T3S applies cyclically bottomup on a prosodic structure that is distinct from the syntactic structure of an expression. In this section, I pursue the hypothesis that the prosodic structure on which T3S applies is matched from the syntactic structure of an expression, along the lines of the Match Theory of syntactic-prosodic constituency correspondence (Selkirk 2011). The Match Theory assumes distinctness of syntactic and prosodic structures. The prosodic structure takes the form of hierarchical constituents that in the default case coincide with the syntactic constituency via a set of Match constraints. What are relevant to this paper are the Match Phrase constraints in (14).

\section{(14) a. MAтсн(XP, $\varphi)$}

The left and right edges of a lexical phrasal projection (XP) in the syntactic structure must correspond to the left and right edges of a phonological phrase $(\varphi)$ in the prosodic structure.

b.

The left and right edges of a phonological phrase $(\varphi)$ in the prosodic structure must correspond to the left and right edges of a lexical phrasal projection (XP) in the syntactic structure.

The Match Theory is a retreat from Selkirk's (1986) Align-XP model which assumes that in the default case only one edge of a syntactic constituent will align with a prosodic boundary. The major empirical evidence in favor of the Match Theory has come from languages such as Connemara Irish in which both edges of a phonological phrase can be detected in the phonology (Elfner 2012, 2015).

I argue that in Mandarin both edges of a phonological phrase are detectable. This is because left- and right-branching expressions show distinct sandhi patterns compared to grammatically unstructured strings, indicating that both the right edges of a left-branching structure and the left edges of a right-branching structure are detectable in the phonology.

The Match constraints are violable. The correspondence between the syntactic and prosodic structure can be altered on a language-particular basis via the intervention of prosodic markedness constraints. Selkirk's (2011) Strong Start constraint, defined in (15), predicts a left-/right-branching asymmetry: 
Because a phonological phrase is higher in the prosodic hierarchy than a prosodic word $(\omega)$, the left-branching prosodic structure in (16a), where every prosodic constituent begins with a phonological phrase or a prosodic word that is immediately followed by a prosodic word, satisfies StRong START. However, the right-branching prosodic structure in (16b) incurs two violations of Strong Start, because both $\varphi 1$ and $\varphi 2$ begin with a prosodic word that is immediately followed by a phonological phrase.

\section{(15) Strong Start}

A prosodic constituent optimally begins with a leftmost daughter constituent not lower in the prosodic hierarchy than the constituent that immediately follows.

(16)

\begin{tabular}{|c|c|}
\hline $\begin{array}{c}\text { a. Left-branching structure: } \\
\text { STRONG START is satisfied }\end{array}$ & $\begin{array}{c}\text { b. Right-branching structure: } \\
\text { STRONG START is violated }\end{array}$ \\
\hline$\varphi 1$ & \\
$\varphi 2 \omega$ & $\omega$ \\
$\omega$ & $\omega$
\end{tabular}

I propose to account for the left-/right-branching asymmetry in T3S by the interaction of the Match Phrase constraints in (14), which require the prosodic structure to be isomorphic to the syntactic structure, and Strong Strong Start, defined in (17), which is a more restricted version of Strong Start.

\section{(17) Strong Strong Start}

A prosodic constituent optimally begins with a leftmost daughter constituent not lower in the prosodic hierarchy than any sister constituent that follows.

The effect of Strong Strong Start is that a right-branching syntactic structure is altered to a prosodic structure that has equal-sisters constituents in the sense of Myrberg (2013), either by flattening the recursive syntactic structure to create a flat, equal-sisters prosodic structure, as in (18a), or by grouping syntactic nonsisters at the left edge to create a recursive, equal-sisters prosodic structure, as in (18b). STrong Strong Start is a more restricted version of Strong StART, because the prosodic structure in (18c), which has unequalsisters constituents, violates Strong Strong Start but satisfies Strong Start.

\section{(18)}

\begin{tabular}{|c|c|c|}
\hline $\begin{array}{c}\text { a. Flat, equal-sisters structure: } \\
\text { Strong Strong StART } \\
\text { is satisfied } \\
\end{array}$ & $\begin{array}{c}\text { b. Recursive, equal-sisters structure: } \\
\text { STRONG STRONG START } \\
\text { is satisfied }\end{array}$ & $\begin{array}{l}\text { c. Unequal-sisters structure: } \\
\text { STRONG STRONG START } \\
\text { is violated } \\
\end{array}$ \\
\hline $\begin{array}{llll}\omega & \omega & \omega & \omega\end{array}$ & $\wedge_{\omega}^{\varphi} \quad \omega_{\omega} \quad \omega_{\omega}$ & $\overbrace{\omega=}^{\varphi 1}$ \\
\hline
\end{tabular}

I propose that Strong Strong Start is ranked variably with respect to the Match Phrase constraints: at slow speech, Strong Strong Start is outranked by the Match Phrase constraints; at fast speech, Strong Strong Start outranks the Match Phrase constraints; and it is also possible that Strong Strong Start and the Match Phrase constraints are equally ranked. Recall that the expression in (19), which has a rightbranching syntactic structure, has two possible sandhi patterns that correlate with speech rate. I suggest that 
these sandhi patterns are derived from different prosodic structures. Specifically, at slow speech, the alternating sandhi pattern is derived when T3S applies cyclically bottom-up on a right-branching prosodic structure that satisfies both $\mathrm{MAtch}(\mathrm{XP}, \varphi)$ and $\mathrm{MAtch}(\varphi, \mathrm{XP})$ but violates Strong Strong Start, as shown in (19a); at fast speech, the non-alternating sandhi pattern is derived when T3S applies on a flat, equal-sisters prosodic structure that violates Мatch $(X P, \varphi)$ but satisfies Strong Strong Start, as shown in (19b). Note that I assume the top node of the prosodic structures is an intonational phrase $(1)$, and the terminal nodes are prosodic words.

$$
\begin{aligned}
& \text { 'buy good wine' } \\
& \text { UR: [ [v mai3 [NP hao3 jiu3] } \\
& \text { buy good wine }
\end{aligned}
$$

a. SR: $\left.\left(\begin{array}{lllll}1 & (\varphi 1 & 3 & (\varphi 2 \mathrm{~s} & 3\end{array}\right)\right)$

\begin{tabular}{|l:l|l|}
\hline Match $(\mathrm{XP}, \varphi)$ & MATch $(\varphi, \mathrm{XP})$ & Strong Strong Start \\
\hline & & $\varphi 1$ \\
\hline
\end{tabular}

(slow speech)

b. SR: $\quad\left(\begin{array}{lllll}1 & \left(\varphi_{1} s\right. & s & 3\end{array}\right)$

\begin{tabular}{|l|l:l|}
\hline Strong Strong Start & Match $(\mathrm{XP}, \varphi)$ & Match $(\varphi, \mathrm{XP})$ \\
\hline & NP & \\
\hline
\end{tabular}

(fast speech)

The right-branching expression in (20) has three possible sandhi patterns that are derived from different prosodic structures. When Strong Strong Start is outranked by the Match Phrase constraints, the output prosodic structure is isomorphic to the syntactic structure, in spite of two violations of Strong Strong Start, as shown in (20a). When Strong Strong Start and the Match Phrase constraints are equally ranked, the output prosodic structures in (20b-c) are partially flattened; one violation of Strong Strong Start is obviated and one violation of Match $(\mathrm{XP}, \varphi)$ is incurred. Finally, when Strong Strong Start outranks the Match Phrase constraints, the output prosodic structures in (20d-e) satisfy Strong Strong StarT; but by flattening the recursive syntactic structure to create a flat, equal-sisters structure, as in (20d), two violations of $\operatorname{Match}(X P, \varphi)$ are incurred, and by grouping syntactic non-sisters at the left edge to create a recursive, equalsisters prosodic structure, as in (20e), one violation of $\operatorname{MAтcH}(X P, \varphi)$ and one violation of $\operatorname{MATcH}(\varphi, X P)$ are incurred. One might assume that (20d) is preferred over (20e) when МАтсн( $\varphi$, XP) outranks Мatch (XP, $\varphi)$, while (20e) is preferred over (20d) when $\operatorname{MATch}_{\text {XP, }} \varphi$ ) outranks МАтсн( $(\varphi$, XP).

$$
\begin{aligned}
& \text { 'want to buy good wine' }
\end{aligned}
$$

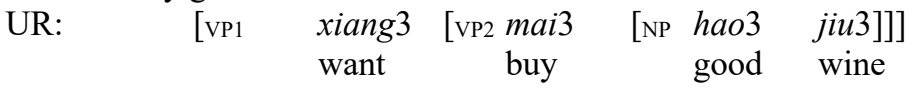

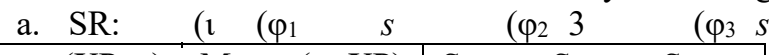

\begin{tabular}{|l:l|l|}
\hline $\operatorname{Match}(X P, \varphi)$ & $\operatorname{Match}(\varphi, X P)$ & Strong Strong Start \\
\hline & & $\varphi_{1}, \varphi_{2}$ \\
\hline
\end{tabular}

Strong Start

$\varphi_{1}, \varphi_{2}$

b. SR: $\quad\left(\begin{array}{llll}1 & \left(\varphi_{1}\right. & 3 & \left(\varphi_{2} s\right.\end{array}\right.$

3)))

\begin{tabular}{|l:l:l|}
\hline MATCh $(X P, \varphi)$ & MAtch $(\varphi$, XP) & Strong Strong StART \\
\hline NP & & $\varphi_{1}$ \\
\hline
\end{tabular}

\section{STRONG START}

$\varphi_{1}$

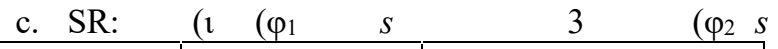

\begin{tabular}{|l|l|l|}
\hline Match $(X P, \varphi)$ & Match $(\varphi, X P)$ & Strong Strong Start \\
\hline VP2 & $\varphi_{1}$ \\
\hline
\end{tabular}

\begin{tabular}{|c|c|c|}
\hline$(\varphi$ & $s$ & 3)) \\
\hline STRONG STRONG START & МАтсн $(\mathrm{XP}, \varphi)$ & $\overline{\text { МАтсн }(\varphi, X P)}$ \\
\hline & $\mathrm{VP} 2, \mathrm{NP}$ & \\
\hline
\end{tabular}

3)))

STRONG START

d. SR:

\begin{tabular}{|c|c|c|}
\hline$\left(\varphi_{1}\left(\begin{array}{ll}\varphi_{2} & s\end{array}\right.\right.$ & $\left(\varphi_{3} s\right.$ & 3))) \\
\hline StRONG STRONG START & МАтсн $(X P, \varphi)$ & МАтсн $(\varphi$, XP) \\
\hline & VP2 & $\varphi_{2}$ \\
\hline
\end{tabular}

e. SR: (1) 
Note that the same sandhi pattern is derived from the prosodic structures in (20c) and (20e), and it is the same as the alternating sandhi pattern derived from the prosodic structure that is isomorphic to the syntactic structure, as in (20a). The prosodic structure in (20c) is one possible output when Strong Strong Start and the Match Phrase constraints are equally ranked. The other possible output, the prosodic structure in $(20 \mathrm{~b})$, gives rise to a distinct sandhi pattern. Because the prosodic structures in (20b) and (20c) have the same violations, and the prosodic structure in $(20 \mathrm{~b})$ is independently attested, I assume the prosodic structure in (20c) is also a possible output. The prosodic structure in (20e) is independently attested, as argued by Shih $(1986,1997)$ and Chen (2000) (see section 2 for relevant discussion.)

I argue that the various possible sandhi patterns for a right-branching expression provide support for Strong Strong Start, which is a more restricted version of Strong Start. The interaction of the Match Phrase constraints and Strong Strong Start predicts that the prosodic structures in (20b) and (20d) are both possible outputs; this is important, because these prosodic structures give rise to distinct sandhi patterns that are possible for the right-branching expression in (20). Note that the prosodic structure in (20c) violates Strong Strong Start but satisfies Strong Start. If the active constraints were the Match Phrase constraints and Strong Start (instead of Strong Strong Start), the prosodic structure in (20c) would be preferred over the prosodic structures in (20b) and (20d), which undergenerates, because two of the possible sandhi patterns for the expression in (20) must be derived from the prosodic structures in (20b) and (20d), respectively.

Finally, recall that expressions that have a left-branching syntactic structure only have a non-alternating sandhi pattern, as exemplified by (21). This lack of variation is expected because the left-branching prosodic structure in (21a) satisfies both the Match Phrase constraints and Strong Strong START; thus, it is the optimal output regardless of the constraint ranking.

(21) 'It is good to leave a bit earlier.'

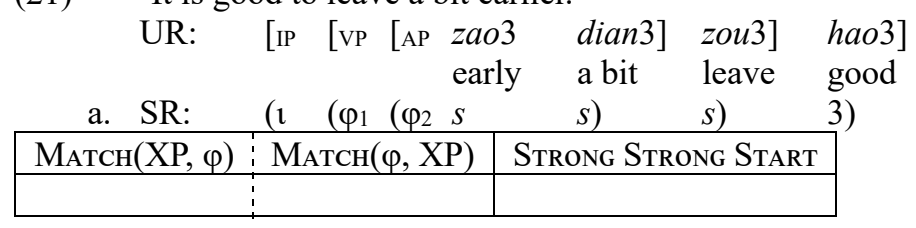

\begin{tabular}{|c:c:c|}
\hline Match $(\mathrm{XP}, \varphi)$ & MAtch $(\varphi, \mathrm{XP})$ & Strong Strong Start \\
\hline & & \\
\hline
\end{tabular}

\begin{tabular}{|c|c|c|}
\hline Strong Strong Start & МАTсH $(X P, \varphi)$ & МАтсн $(\varphi$, XP) \\
\hline & & \\
\hline $\begin{array}{ll}\text { b. SR: } \\
\text { c. SR: }\end{array}$ & $\begin{array}{l}* s \\
* 3\end{array}$ & $\begin{array}{l}s \\
s\end{array}$ \\
\hline
\end{tabular}

To summarize, I have proposed a Match-theory analysis of the left-/right-branching asymmetry in T3S. Specifically, the interaction of the Match Phrase constraints and Strong Strong Start predicts that different prosodic structures are possible outputs for expressions that have a right-branching syntactic structure, while for expressions that have a left-branching syntactic structure the only possible output is a left-branching prosodic structure. The various possible sandhi patterns for a right-branching expression and the nonalternating sandhi pattern for a left-branching expression can be derived when T3S applies cyclically bottomup on the proposed prosodic structures. In the next section, I will provide an OT analysis of the T3S process that is compatible with the prosodic structures proposed in this section.

\section{The Tone 3 Sandhi process}

As mentioned previously, I assume, following Yip $(1980,2002)$, that T3 is underlyingly L. In addition, I will assume, following Duanmu (1990 et seq.), that the Mandarin tones have the underlying representations in (22) - in particular that a tone-bearing syllable forms a moraic trochee, where the head mora is on the left, and that the tone-bearing unit is the mora. Note that a sandhi tone has the phonological characteristics of T2. 
(22) Underlying representation of tones in Mandarin

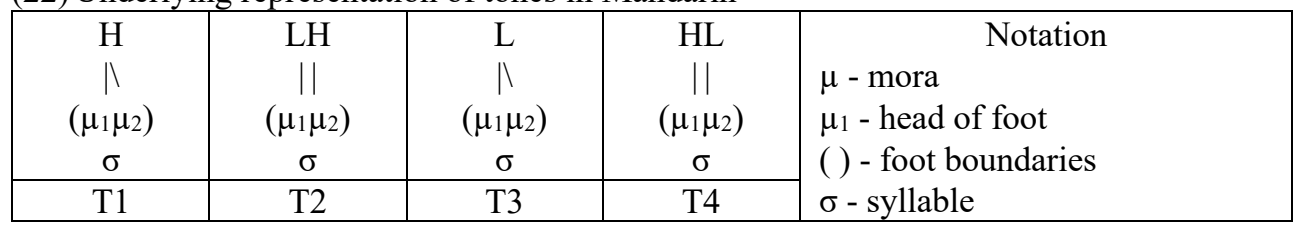

The analysis I will develop in this section builds on Kuo, Xu and Yip's (2007) proposal that T3S, where a H tone is inserted between two $\mathrm{L}$ tones, is a particular repair of the OCP-L violation incurred by two successive T3 syllables. They propose to rule out the unattested repairs by appropriate ranking of the constraints in (23).

\section{(23)a. OCP-L}

Adjacent $\mathrm{L}$ tones are prohibited in the output.

b. Max-T

Every tone in the input must have a correspondent in the output.

\section{c. DEP-T}

Every tone in the output must have a correspondent in the input.

\section{d. *Head/L}

The head of a prosodic constituent is not a $\mathrm{L}$ tone.

The tableau in (24) illustrates Kuo, Xu and Yip's analysis of the T3S process. With OCP-L outranking DeP$T$, the attested output in (24a) is preferred over the faithful output in (24d). The outputs in (24b-c) are ruled out because they violate MAX-T or *Head/L in addition to Dep-T. In particular, the output in (24c) is ruled out because it is assumed that the head of the foot (underlined) is the syllable to the left.

(24)

\begin{tabular}{|l|l|l|l|l|}
\hline$/$ L.L/ & OCP-L & MAX-T & DeP-T & *HEAD/L \\
\hline a. $(\underline{\text { LH}} . \mathrm{L})$ & & & $*$ & \\
\hline b. $(\underline{\mathrm{H}} . \mathrm{L})$ & & $* !$ & $*$ & \\
\hline c. $(\underline{\mathrm{L}} . \mathrm{HL})$ & & & $*$ & $* !$ \\
\hline d. (L.L) & $* !$ & & & $*$ \\
\hline
\end{tabular}

Because OCP-L will penalize any adjacent $\mathrm{L}$ tones in the output, other tone combinations where adjacent $\mathrm{L}$ tones occur must also be considered. In particular, the analysis must also explain why a $\mathrm{T} 3$ is unchanged when it is followed by a T2 or a sandhi tone. Kuo, Xu and Yip confront this problem by positing that T2 is underlyingly MH rather than LH. They also argue that Dep-T must outrank *HeAd/L in order to account for the fact that no change is observed when a T3 is followed by a T2, as illustrated by the tableau in (25).

$(25)$

\begin{tabular}{|l|l|l|l|l|}
\hline$/$ L.MH/ & OCP-L & MAX-T & DEP-T & *HEAD/L \\
\hline a. $(\underline{\text { L.MH}})$ & & & & $* !$ \\
\hline b. (느.MH) & & & $* !$ & \\
\hline
\end{tabular}

However, Kuo, Xu and Yip neglect the fact that a T3 also remains $\mathrm{L}$ when it is followed by a sandhi tone. Given their account of the T3S process, a sandhi tone is LH rather than MH. Thus, the fact that [L.LH(.L)] outputs are possible in spite of violations of the top-ranked OCP-L is not accounted for.

One might take a different approach and assume a more specific version of OCP that only penalizes adjacent T3 syllables in the output. But I argue with Kuo, Xu and Yip that Mandarin observes the more general OCP-L constraint, because when more than two successive T3 syllables occur in a prosodic constituent, all but the rightmost $\mathrm{T} 3$ is changed into the sandhi tone. For example, Kuo, $\mathrm{Xu}$ and Yip consider the outputs in (26a-d) for the input /L.L.L/, and argue that the output in (26b), in which only the syllable in the middle is changed into a sandhi tone, must be ruled out by the general OCP-L constraint. In addition, to rule out the output in (26c), Max-T must outrank Dep-T. However, Kuo, Xu and Yip do not consider the 
output in (26e), in which a $\mathrm{H}$ tone is inserted into the rightmost syllable. Recall that in (24), the output [L.HL] is ruled out by ${ }^{*} \mathrm{HEAD} / \mathrm{L}$ because it is assumed that the head of the foot is the syllable to the left. The output in (26e), however, does not violate ${ }^{*} \mathrm{HEAD} / \mathrm{L}$ because the syllable in the middle is not the head of the foot.

(26)

\begin{tabular}{|c|c|c|c|c|}
\hline /L.L.L/ & OCP-L & Max-T & Dep-T & *HEAD/L \\
\hline $\mathrm{a} .($ LH.LH.L) & & & $* *$ & \\
\hline b. (L.LH.L) & $* !$ & & $*$ & * \\
\hline c. (L.H.L) & & $* !$ & * & * \\
\hline d. (L.L.L) & $* * !$ & & & \\
\hline e. (LH.L.HL) & & & $* *$ & \\
\hline
\end{tabular}

To summarize, Kuo, Xu and Yip's analysis needs to be modified in order to account for the fact that [L.LH(.L)] outputs are possible when a T3 is followed by (a T2 or) a sandhi tone and to rule out repairs of OCP-L violations in which a $\mathrm{H}$ tone is inserted into the syllable to the right, resulting in [... L.HL (...)], when more than two successive T3 syllables occur in the input.

I propose that when a $\mathrm{T} 3$ is followed by a $\mathrm{T} 2$ or a sandhi tone in the input, the $\mathrm{L}$ tone of the $\mathrm{T} 2$ or the sandhi tone is deleted and the $\mathrm{L}$ tone of the T3 spreads onto the head mora of the following syllable. In this way, OCP-L is obeyed and the input tonal feature value of each mora is also preserved. ${ }^{1}$ This process is illustrated in (27). ${ }^{2}$

(27)

\begin{tabular}{|cc|c|}
\hline \multicolumn{2}{|c|}{ a. Input: OCP-L is violated } & b. Output: OCL-L is obeyed \\
\hline $\mathrm{L}_{1}$ & $\mathrm{~L}_{2} \mathrm{H}$ & $\mathrm{L}_{1} \quad \mathrm{H}$ \\
$\|$ & $\|$ & $/|\backslash|$ \\
$\left(\mu_{1} \mu_{2}\right)$ & $\left(\mu_{1} \mu_{2}\right)$ & $\left(\mu_{1} \mu_{2}\right)\left(\mu_{1} \mu_{2}\right)$ \\
$\mathrm{T} 3$ & $\mathrm{~T} 2$ & $\mathrm{~T} 3 \quad \mathrm{~T} 2$ \\
\hline
\end{tabular}

To derive the output in (27b) from the input in (27a), Dep-T must outrank MAX-T to block the unattested output [LH.LH], in which a $\mathrm{H}$ tone is inserted between the two L tones. However, with Dep-T outranking Max-T, the unattested output [L.H.L] would be preferred over the attested output [LH.LH.L] for the input /L.L.L/. To confront this problem, I posit that MAx-L $\mu \mu$, which militates against deletion of the L tone of a T3, outranks DEP-T.

\section{(28) Max-L $\mu \mu$}

Every L tone that is linked to two moras in the input must have a correspondent in the output.

To generally rule out repairs of OCP-L violations in which a $\mathrm{H}$ tone is inserted into the syllable to the right, as in [L.HL], I propose to replace Kuo, Xu and Yip's *HEAD/L constraint with a positional faithfulness constraint, HD-ID-T, defined in (29). With HD-ID-T and the assumption that the head mora of a syllable is to the left, the inserted $\mathrm{H}$ tone in T3S must be link to the non-head mora of the syllable to the left.

\section{(29) Ho-ID-T}

The head mora of a syllable must have the same tonal feature value in the input and the output.

I propose the following ranking for the constraints active in the analysis:

\footnotetext{
${ }^{1}$ I will assume that the output in (27b) does not violate HD-ID-T, defined in (29), because the head mora of the T2 syllable has the same tonal feature value in the input and the output, although it is not associated with the same tonal autosegment in the input and the output. Whether constraints such as HD-ID-T should be defined on the feature level or autosegmental level is a broader question that the paper does not address.

${ }^{2}$ Possible evidence in favor of the output in (27b) can be found in the pitch track of T3-T2 from Xu (1997: 75), where the L tone of the T3 syllable is delayed to the middle of the following T2 syllable (Michael Kenstowicz, p.c.).
} 


\section{(30) OCP-L, MAx-L $\mu \mu$ > DEP-T >> MAX-T, HD-ID-T}

The tableau in (31) illustrates the analysis of the T3S process. The attested output in (31a) is preferred over the output in (31b) because the latter violates HD-ID-T. The output in (31c) and the faithful output (31d) are ruled out because they violate the top-ranked MAX-L $\mu \mu$ and OCP-L, respectively.

\begin{tabular}{|c|c|c|c|c|c|}
\hline $\begin{array}{cc}\mathrm{L}_{1} & \mathrm{~L}_{2} \\
\| & \| \\
\left(\mu_{1} \mu_{2}\right) & \left(\mu_{1} \mu_{2}\right) \\
\mathrm{T} 3 & \mathrm{~T} 3\end{array}$ & OCP-L & MAX-L $\mu \mu$ & DEP-T & Max-T & HD-ID-T \\
\hline $\begin{array}{ccc}\mathrm{L}_{1} \mathrm{H} & \mathrm{L}_{2} \\
\| & \| \\
\left(\mu_{1} \mu_{2}\right) & \left(\mu_{1} \mu_{2}\right) \\
\mathrm{T} 2 & \mathrm{~T} 3\end{array}$ & & & $*$ & & \\
\hline $\begin{array}{lcc}\text { b. } & \mathrm{L}_{1} & \mathrm{HL}_{2} \\
& \wedge & \| \\
& \left(\mu_{1} \mu_{2}\right) & \left(\mu_{1} \mu_{2}\right) \\
& \mathrm{T} 2 & \mathrm{~T} 3 \\
\end{array}$ & & & * & & $* !$ \\
\hline \begin{tabular}{ccc} 
c. & \multicolumn{2}{c}{$\mathrm{L}_{1}$} \\
& $/|| \mid$ \\
$\left(\mu_{1} \mu_{2}\right)$ & $\left(\mu_{1} \mu_{2}\right)$ \\
& $\mathrm{T} 3$ & $\mathrm{~T} 3$ \\
\end{tabular} & & $* !$ & & $*$ & \\
\hline $\begin{array}{ccc}\text { d. } & \mathrm{L}_{1} & \mathrm{~L}_{2} \\
& \bigwedge & \Lambda \\
& \left.\mu_{1} \mu_{2}\right) & \left(\mu_{1} \mu_{2}\right) \\
& \mathrm{T} 3 & \mathrm{~T} 3\end{array}$ & $* !$ & & & & \\
\hline
\end{tabular}

The tableau in (32) considers an input in which more than two successive T3 syllables occur. The attested output in (32a), in which all but the rightmost $\mathrm{T} 3$ is changed into the sandhi tone, is preferred over the output in (32b), because the latter violates HD-ID-T. The output in (32c), in which only the syllable in the middle is changed into a sandhi tone, is ruled out by Max-L $\mu \mu$. The faithful output in (32d) is ruled out by OCP-L.

\begin{tabular}{|c|c|c|c|c|c|}
\hline 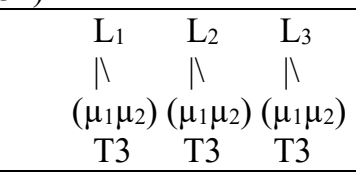 & OCP-L & MAX-L $\mu \mu$ & DeP-T & MAX-T & HD-ID-T \\
\hline $\begin{array}{ccc}\mathrm{L}_{1} \mathrm{H} & \mathrm{L}_{2} \mathrm{H} & \mathrm{L}_{3} \\
\| & \| & \| \\
\left(\mu_{1} \mu_{2}\right) & \left(\mu_{1} \mu_{2}\right) & \left(\mu_{1} \mu_{2}\right) \\
\mathrm{T} 2 & \mathrm{~T} 2 & \mathrm{~T} 3\end{array}$ & & & ** & & \\
\hline $\begin{array}{cccc}\text { b. } & \mathrm{L}_{1} \mathrm{H} & \mathrm{L}_{2} & \mathrm{HL}_{3} \\
\| & \| & \| \\
& \| & \| \\
\left(\mu_{1} \mu_{2}\right) & \left(\mu_{1} \mu_{2}\right) & \left(\mu_{1} \mu_{2}\right) \\
\mathrm{T} 2 & \mathrm{~T} 2 & \mathrm{~T} 3\end{array}$ & & & $* *$ & & $* !$ \\
\hline \begin{tabular}{cccc} 
c. & $\mathrm{L}_{1}$ & $\mathrm{H}$ & $\mathrm{L}_{3}$ \\
$/ \mid$ & $\backslash$ & $\|$ \\
& \multicolumn{1}{c}{$\left(\mu_{1} \mu_{2}\right)$} & $\left(\mu_{1} \mu_{2}\right)$ & $\left(\mu_{1} \mu_{2}\right)$ \\
$\mathrm{T} 3$ & $\mathrm{~T} 2$ & $\mathrm{~T} 3$
\end{tabular} & & $* !$ & * & * & \\
\hline $\begin{array}{cccc}\text { d. } & \mathrm{L}_{1} & \mathrm{~L}_{2} & \mathrm{~L}_{3} \\
& \bigwedge & \bigwedge & \bigwedge \\
& \left.\mu_{1} \mu_{2}\right) & \left(\mu_{1} \mu_{2}\right) & \left(\mu_{1} \mu_{2}\right) \\
& \mathrm{T} 3 & \mathrm{~T} 3 & \mathrm{~T} 3\end{array}$ & *!* & & & & \\
\hline
\end{tabular}


Finally, the proposed analysis must be able to derive an alternating sandhi pattern when T3S applies cyclically bottom-up on a right-branching prosodic structure, and derive a non-alternating sandhi pattern when T3S applies cyclically bottom-up on a left-branching prosodic structure. The tableau in (33) considers an input in which the syllable in the middle has been changed into a sandhi tone in the first cycle when T3S applies cyclically bottom-up on a right-branching prosodic structure. The attested output in (33a), in which the $\mathrm{L}$ tone of the sandhi tone in the middle is deleted and the $\mathrm{L}$ tone of the T3 syllable to the left spreads onto the head mora of the following syllable, gives rise to an alternating sandhi pattern; it is preferred over the output in (33b), which gives rise to a non-alternating sandhi pattern via insertion of an $\mathrm{H}$ tone, and the faithful output in (33c), because the unattested outputs violate DEP-T and OCP-L respectively, both of which outrank Max-T.

(33)

\begin{tabular}{|c|c|c|c|c|c|}
\hline $\begin{array}{ccc}\mathrm{L}_{1} & \mathrm{~L}_{2} \mathrm{H} & \mathrm{L}_{3} \\
\| & \| & \wedge \\
\left(\mu_{1} \mu_{2}\right) & \left(\mu_{1} \mu_{2}\right) & \left(\mu_{1} \mu_{2}\right) \\
\mathrm{T} 3 & \mathrm{~T} 2 & \mathrm{~T} 3\end{array}$ & OCP-L & MAX-L $\mu \mu$ & Dep-T & Max-T & HD-ID-T \\
\hline 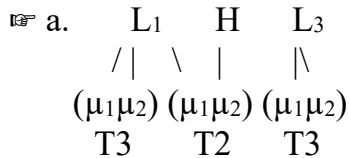 & & & & * & \\
\hline $\begin{array}{cccc}\text { b. } & \mathrm{L}_{1} \mathrm{H} & \mathrm{L}_{2} \mathrm{H} & \mathrm{L}_{3} \\
\| & \| & \| \\
& \| & \| & \| \\
\left(\mu_{1} \mu_{2}\right) & \left(\mu_{1} \mu_{2}\right) & \left(\mu_{1} \mu_{2}\right) \\
& \mathrm{T} 2 & \mathrm{~T} 2 & \mathrm{~T} 3 \\
\end{array}$ & & & $* !$ & & \\
\hline $\begin{array}{cccc}c . & \mathrm{L}_{1} & \mathrm{~L}_{2} \mathrm{H} & \mathrm{L}_{3} \\
& \Lambda & \| & \| \\
& \left(\mu_{1} \mu_{2}\right) & \left(\mu_{1} \mu_{2}\right) & \left(\mu_{1} \mu_{2}\right) \\
& \mathrm{T} 3 & \mathrm{~T} 2 & \mathrm{~T} 3\end{array}$ & $* !$ & & & & \\
\hline
\end{tabular}

It is easy to see that if the output in (33a) enters into another cycle along with a T3 syllable to the left, as in (34a), T3S will apply between the two successive T3 syllables, and a $\mathrm{H}$ tone will be inserted into the syllable to the left, as in (34b).

(34)

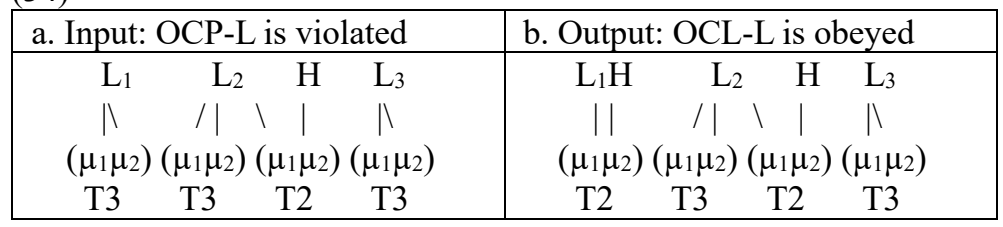

When T3S applies cyclically bottom-up on a left-branching prosodic structure, in the first cycle the leftmost syllable will be changed into a sandhi tone. In the next cycle, T3S will apply between the two successive T3 syllables in (35a), and a $\mathrm{H}$ tone will be inserted into the syllable to the left, as in (35b). This process gives rise to a non-alternating sandhi pattern.

(35)

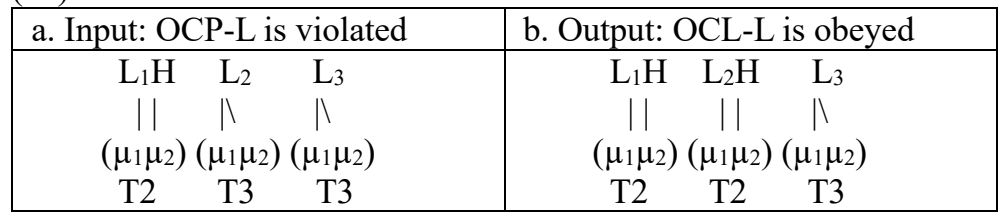

To summarize, I have provided an OT analysis of the T3S process that is compatible with the prosodic structures proposed in section 3. The proposed analysis ensures that when more than two successive T3 
syllables occur in a flat prosodic constituent, all but the rightmost T3 is changed into the sandhi tone. In addition, the proposed analysis successfully derives an alternating sandhi pattern when T3S applies cyclically bottom-up on a right-branching prosodic structure, and derives a non-alternating sandhi pattern when T3S applies cyclically bottom-up on a left-branching prosodic structure.

\section{Conclusion}

This paper provided an analysis of the left-/right-branching asymmetry in Mandarin T3S: while expressions that have a left-branching syntactic structure only have a non-alternating sandhi pattern in which all but the rightmost $\mathrm{T} 3$ is changed to the sandhi tone, for expressions that have a right-branching syntactic structure various sandhi patterns are possible. I pursued the hypothesis that $\mathrm{T} 3 \mathrm{~S}$ applies cyclically bottom-up on a prosodic structure matched from the syntactic structure of an expression, along the lines of the Match Theory of syntactic-prosodic constituency correspondence (Selkirk 2011). Specifically, the interaction of Match Phrase constraints and Strong Strong StarT, which is a more restricted version of Selkirk's (2011) StRong START constraint, predicts that different prosodic structures are possible outputs for a right-branching expression, while for a left-branching expression the only possible output is a left-branching prosodic structure. Then, I showed that the various possible sandhi patterns for a right-branching expression and the non-alternating sandhi pattern for a left-branching expression can be derived when T3S applies cyclically bottom-up on the proposed prosodic structures.

\section{References}

Chao, Yuen Ren. 1930. A System of Tone Letters. Le Maitre Phonetique 30: 24-27.

Chao, Yuen Ren. 1948. Mandarin Primer. Cambridge, MA: Harvard University Press.

Chao, Yuen Ren. 1968. A Grammar of Spoken Chinese. Berkeley, CA: University of California Press.

Chen, Matthew Y. 2000. Tone Sandhi: Patterns across Chinese Dialects. Cambridge, MA: Cambridge University Press.

Cheng, Chin-Chuan. 1970. Domains of phonological rule application. In Studies Presented to Robert B. Lee by his students, 39-60. Edmonton: Linguistic Research.

Cheng, Chin-Chuan. 1973. A synchronic phonology of Mandarin Chinese. The Hague: Mouton.

Duanmu, San. 1990. A formal study of syllable, tone, stress and domain in Chinese languages. PhD dissertation, MIT.

Elfner, Emily. 2012. Syntax-prosody interactions in Irish. PhD dissertation, University of Massachusetts, Amherst.

Elfner, Emily. 2015. Recursion in prosodic phrasing: Evidence from Connemara Irish. Natural Language \& Linguistic Theory 33: 1169-1208.

Kaisse, Ellen. M. 1985. Connected Speech: The Interaction of Syntax and Phonology. Orlando, FL: Academic Press, Inc.

Kuo, Yu-Ching, Xu, Yi, and Yip, Moira. 2007. The phonetics and phonology of apparent cases of iterative tonal change in Standard Chinese. Tones and tunes, (2), 211-237.

Myrberg, Sara. 2013. Sisterhood in prosodic branching. Phonology, 30(1), 73-124.

Selkirk, Elisabeth. 1986. On derived domains in sentence phonology. Phonology Yearbook 3: 371-405.

Selkirk, Elisabeth. 2011. The syntax-phonology interface. In The Handbook of Phonological Theory (2nd edition), edited by J. Goldsmith, J. Riggle and A. Yu, 435-484.

Shih, Chi-Lin. 1986. The Prosodic Domain of Tone Sandhi in Chinese. PhD dissertation, University of California, San Diego.

Shih, Chi-Lin. 1997. Mandarin third tone sandhi and prosodic structure. In Studies in Chinese Phonology, edited by Jialing Wang and Norval Smith, 81-123. Berlin: Mouton de Gruyter.

$\mathrm{Xu}$, Yi. 1997. Contextual tonal variations in Mandarin. Journal of Phonetics 25: 61-83.

Yip, Moira. 1980. The Tonal Phonology of Chinese. PhD dissertation, MIT.

Yip, Moira. 2002. Tone. Cambridge: Cambridge University Press. 